\title{
Caminhos da internacionalização dos periódicos de saúde coletiva
}

\author{
Pathways for the internationalization of public health journals
}

\author{
José Leopoldo Ferreira Antunes', Aluísio Jardim Dornellas de Barros², Maria Cecília de Souza \\ Minayo ${ }^{3}$
}

DOI: $10.1590 / 0103-1104201912217$

RESUMO Esse texto teve como objetivo apresentar caminhos para que o meio editorial em saúde coletiva incremente a visibilidade das revistas brasileiras no exterior. Sinteticamente, essas medidas são: publicação dos artigos em inglês; indexação dos periódicos nas principais bases de dados internacionais (PubMed, Scopus, Web of Science etc.); inclusão das revistas nos repositórios on-line internacionais (SciELO, PMC, Europe PMC etc.); adoção de sistema de gestão editorial em inglês, permitindo a comunicação entre autores, editores e revisores de diferentes países; adoção de licença de direitos de cópia, permitindo a divulgação dos artigos publicados em repositórios individuais e institucionais. Muitas dessas sugestões já vêm sendo implantadas, e várias revistas no Brasil já avançaram no trajeto assinalado. Essas diretrizes implicam custos adicionais e empenho editorial. A produção de uma revista científica com impacto internacional não deveria ser responsabilidade apenas dos editores e dos autores. É fundamental que as agências de fomento à pesquisa criem fontes sustentáveis de financiamento e de internacionalização para as revistas brasileiras de reconhecido mérito.

PALAVRAS-CHAVE Publicações periódicas. Resumos e indexação como assunto. Saúde global.

1 Universidade de São Paulo (USP), Faculdade de Saúde Pública - São Paulo (SP), Brasil.

leopoldo@usp.br

2 Universidade Federal de Pelotas (UFPEL) - Programa de Pós-Graduação em Epidemiologia - Pelotas (RS), Brasil.

${ }^{3}$ Fundação Oswaldo Cruz (Fiocruz), Escola Naciona de Saúde Pública Sergio Arouca (Ensp) - Rio de Janeiro (RJ), Brasil.
ABSTRACT The objective of this text was to explore how public health journals edited in Brazil can increase their international impact. Synthetically, the following measures were proposed: the publication of articles in English; the indexing of journals in the leading international databases (PubMed, Scopus, Web of Science etc.); the inclusion of journals in international online repositories (SciELO, PMC, Europe $P M C$ etc.); the adoption of editorial a management system allowing for the communication in English between authors, editors and reviewers from different countries; the adoption of copyrights allowing for free access of the articles, and their distribution in individual and institutional repositories. Several journals in Brazil have already implemented many of these suggestions. These guidelines imply additional costs and editorial commitment. The production of a scientific journal with international impact should not be the responsibility of editors and authors alone. Governmental agencies for funding research should provide sustainable sources to support the internationalization of Brazilian journals with recognized merit.

KEYWORDS Periodicals. Abstracting and indexing as topic. Global health. 


\section{Introdução}

A ciência (diferentemente da tecnologia hard) não tem reserva de mercado. Ela já nasceu com vocação para se expandir. Seu berço mais expressivo foram os países ocidentais considerados mais desenvolvidos. Neste século, algumas nações emergentes vêm tomando consciência da importância de dar visibilidade internacional ao produto de seu conhecimento, por meio das mais diferentes estratégias. E nesse caminho, muitas questões se fazem presentes: por que internacionalizar? Em que direção?

Em texto publicado na revista 'Nature', Adams ${ }^{1}$ apresentou uma análise dos artigos das três últimas décadas e concluiu que, na atualidade, a melhor ciência provém da colaboração internacional. $\mathrm{O}$ autor avaliou que, no Reino Unido, a proporção de trabalhos com participação de autores de mais de um país aumentou de 20\% para 50\% entre 1981 e 2012. No Brasil, essa proporção oscilou em torno de $25 \%$ em todo o período, com o máximo na década de 1990, quando alcançou pouco mais de $30 \%^{2}$. A publicação feita com a colaboração de vários pesquisadores também impacta positivamente os índices de citação.

Cunha-Mello ${ }^{3}$ fez uma longa revisão sobre como a internacionalização da ciência é pensada e efetivada nos países ditos desenvolvidos, e apresenta dois exemplos: o caso da Dinamarca, e a forma de indução e avaliação do Conselho de Pesquisa Europeu. Quatro atores principais estão implicados em ambos os exemplos: os governos, as agências de fomento, as universidades e os grupos de pesquisa. Nos locais em que a ciência e a tecnologia passaram a fazer parte do bem-estar cotidiano da população, os governantes já entenderam que as sociedades do conhecimento induzem políticas econômicas, sociais e culturais que podem resultar em bem-estar das nações. No entanto, a ciência não pode ser feita apenas com ações pontuais, as quais costumam ajudar, mas não resolvem o âmago da questão da internacionalização, por exemplo, em um país como o Brasil.
No caso brasileiro, Farias 4 considerou que a chave para as revistas brasileiras é a intensificação do conhecimento da língua inglesa e a especialização dos editores, revisores e autores. Para elevar o padrão de compreensão dos textos, os tradutores precisam ser profissionais nativos de países onde o inglês é a língua oficial. Mas para isso é preciso que a editoração científica seja valorizada e devidamente financiada.

O caso aqui em questão é o papel dos periódicos do campo da saúde pública/saúde coletiva, hoje uma das áreas mais atuantes na produção científica nacional. A introdução mais geral sobre internacionalização da ciência se justifica pelo fato de que muitos obstáculos encontrados hoje no caminho pretendido são fruto da falta de uma visão abrangente que contemple profissionalização e financiamento. Nos últimos anos tem havido uma forte indução da Coleção SciELO Brasil para a profissionalização e o aprimoramento dos processos editoriais, e para que as revistas se abram ao público do exterior.

O texto a seguir tem como objetivo apresentar alguns caminhos para que o meio editorial em saúde coletiva incremente a visibilidade das revistas brasileiras no exterior. Muitas sugestões já vêm sendo implantadas, e no Brasil várias revistas já avançaram no trajeto assinalado. Tudo isso certamente tem valor, desde que não se perca a perspectiva mais ampla de valorizar a divulgação da produção nacional. Por esse motivo, muitas questões ficam pendentes, e é sobre elas também que se fazem algumas considerações finais.

\section{Caminhos iniciais da internacionalização dos periódicos}

A história da imprensa científica em ciências da saúde no Brasil remonta à primeira metade do século XIX. Os primeiros periódicos médicos foram criados pela Academia Imperial 
de Medicina - o 'Propagador das Ciências Médicas', em 1827, e o 'Semanário de Saúde Pública', em $1831^{5}$. Tiveram vida curta e foram interrompidos poucos anos depois, por dificuldades financeiras. Mas nas décadas seguintes outros periódicos os sucederam na divulgação das pesquisas de saúde e em assuntos corporativos da classe médica, como é o caso da 'Gazeta Médica da Bahia', 'Brasil Médico', da 'Gazeta Clínica' e da 'Revista Médica de São Paulo', entre outras.

Desde seus primórdios, esses periódicos tiveram a internacionalização como uma diretriz editorial, uma vez que traziam notícias de outros países e traduziam artigos publicados no exterior. Ou seja, a internacionalização era uma interação valorizada pelo meio profissional, mas tinha apenas um sentido 'de lá para cá'.

Especificamente aplicada à área da saúde coletiva, a 'Revista de Saúde Pública'6 é a mais antiga do País, tendo sido lançada em 1967 pela Faculdade de Saúde Pública da Universidade de São Paulo (USP) como sucedânea de outros periódicos, o mais antigo deles criado em 1919. As outras revistas que integram o quadro atual da divulgação científica em saúde coletiva no País foram criadas nas décadas seguintes: 'Saúde em Debate'7 (a revista do Centro Brasileiro de Estudos de Saúde - Cebes), em 1976; os ‘Cadernos de Saúde Pública'8, da Escola Nacional de Saúde Pública Sergio Arouca (Ensp) da Fundação Oswaldo Cruz (Fiocruz), em 1985; 'Epidemiologia e Serviços de Saúde’, mantida pelo Ministério da Saúde desde 1992; os periódicos da Associação Brasileira de Saúde Coletiva (Abrasco) 'Ciência \& Saúde Coletiva'10, em 1996, e a 'Revista Brasileira de Epidemiologia'11, em 1998; além de 'Physis', 'Interface: Comunicação, Saúde e Educação', 'Saúde e Sociedade', 'Cadernos Saúde Coletiva', 'Revista Brasileira de Saúde Ocupacional'. Esta relação não é abrangente e está longe de ser completa. Em 2014, a Abrasco criou o Fórum de Editores de Saúde Coletiva ${ }^{12}$, congregando 26 periódicos da área em todo o País.

Do ponto de vista editorial, a saúde coletiva é uma das áreas mais bem servidas no País.
Além desses periódicos, a produção científica da área ocupa um espaço considerável nas revistas médicas, odontológicas, de nutrição, de enfermagem, de educação física, das ciências da saúde em geral. Ademais, periódicos no exterior publicam artigos escritos no Brasil, qualificando uma interlocução internacional que ultrapassa as revistas sediadas no País.

A multiplicidade e a longevidade da produção bibliográfica em saúde coletiva no Brasil atestam suas muitas funções e utilidades. Os periódicos da área veiculam a comunicação entre estudantes, pesquisadores e trabalhadores de saúde. Além disso, propiciam a interação destes com os gestores dos serviços de saúde e com os formuladores das políticas do setor, diferentemente das áreas em que a comunicação científica é mais restrita ao meio acadêmico. Muitos artigos também são lidos pelo público em geral, porque estão em acesso aberto, e alguns deles, nas redes sociais. $\mathrm{E}$ os conteúdos promovem a educação continuada, a atualização profissional e a inovação em procedimentos, programação e políticas de saúde.

Em todos esses aspectos, o escopo das revistas se beneficia da troca de experiências propiciada pela interação internacional. No contexto mais recente, já não é tão importante divulgar notícias de outros países e traduzir artigos publicados no exterior - a preocupação dos primeiros periódicos de nossa história -, pois os periódicos sediados no exterior circulam extensamente no Brasil. De um lado, há o movimento de acesso aberto à comunicação científica; de outro, o recurso ao portal periodicos.capes.gov.br para acesso a muitas revistas que ficariam restritas aos assinantes e leitores pagantes. Assim, o alvo principal dos esforços de internacionalização dos periódicos de saúde coletiva passou a ser dar visibilidade internacional ao conhecimento científico produzido no País. Ou seja, o sentido 'de lá para cá' de nossa interação foi bem resolvido com o acesso on-line e o acesso livre. Mas a divulgação 'de cá para lá' de nossa atividade profissional ainda enfrenta dificuldades e precisa ser expandida, de 
modo a ampliar a visibilidade internacional do conhecimento aqui produzido.

A perspectiva de divulgar a produção científica do País no exterior favorece a comunicação dos problemas de saúde do País, dos métodos utilizados e das tentativas de solução. A divulgação internacional é também um meio para fortalecer os periódicos nacionais, tornando-os mais atraentes para o meio profissional e oferecendo aos pesquisadores difusão mais ampla de seus estudos.

\section{Caminhos atuais para intensificar a internacionalização}

Uma primeira medida relevante na atualidade é publicar os artigos em inglês. Tem-se valorizado a publicação simultânea em inglês e português como estratégia preferencial para maximizar a divulgação dos artigos tanto no País como no exterior. Essa iniciativa começou a ser aplicada nos anos 1980, e expandiu-se consideravelmente nas décadas seguintes, apesar do custo adicional para os autores e os periódicos. Parte-se da premissa de que o inglês é, inegavelmente, o idioma mais utilizado mundialmente na comunicação científica.

Em geral, estudantes, pesquisadores e profissionais brasileiros são proficientes para a leitura de artigos em inglês. No entanto, poucos conseguem escrever em inglês com eficiência e efetividade, o que gera um custo adicional para a publicação científica. Nesse sentido, a recomendação de verter os artigos para o inglês deveria ser acompanhada pelo apoio financeiro de que as revistas necessitam para cumprir essa tarefa.

Essa é uma questão-chave. No Brasil, de maneira geral, o poder público não valoriza a divulgação científica. Enquanto agências internacionais de fomento pagam os custos de publicação dos artigos gerados pelos projetos que financiam, as agências nacionais fazem o contrário. Por exemplo, o Conselho Nacional de Desenvolvimento Científico e Tecnológico (CNPq) não permite incluir a publicação dos artigos ou livros no orçamento dos projetos. Seria preciso reverter essa tendência.

Uma segunda medida é indexar as revistas editadas no Brasil nas principais bases de dados internacionais: Medline, Literatura Latino-Americana e do Caribe em Ciências da Saúde (Lilacs), Embase, Web of Science e outras. Essa iniciativa também é fundamental, pois os artigos publicados em revistas não indexadas têm circulação muito restrita e é difícil encontrá-los. Cumprir o objetivo de indexação internacional implica custo e esforço dos periódicos, pois eles precisam atingir e manter os critérios de excelência editorial estipulados pelas bases de dados.

Muitos periódicos já foram indexados, o que intensificou a interlocução dos autores no âmbito internacional. A divulgação do resumo de seus artigos nos mecanismos de busca especializada potencializa sua leitura por profissionais interessados nos temas publicados. Além disso, o aumento da participação de revistas brasileiras nas bases de dados que contabilizam citações bibliográficas ajuda a soerguer as demais revistas, pois resulta em melhor avaliação dos indicadores métricos de todos os periódicos, como é o caso do fator de impacto e do índice H. É muito importante que as revistas brasileiras se organizem e se qualifiquem para sua indexação nas bases internacionais.

A terceira proposição para os periódicos de saúde coletiva é sua inclusão nos principais repositórios internacionais. Essa iniciativa também vem sendo progressivamente adotada, e algumas revistas brasileiras já utilizam esse recurso para dinamizar a divulgação internacional dos artigos. Tão logo são publicados em meio físico ou digital, os textos completos passam a ter seus arquivos HTML e PDF distribuídos gratuitamente por esses repositórios, de modo análogo à publicação on-line em Scientific Electronic Library Online (SciELO). Além disso, esses repositórios inserem links de direcionamento nos mecanismos de busca, 
aumentando a visibilidade internacional dos artigos. Para as revistas brasileiras que ainda não o fizeram, a adoção dessa medida também é uma estratégia muito interessante.

Os principais repositórios internacionais são os seguintes:

- PubMed Central (PMC), o acervo com mais de três mil periódicos da National Library of Medicine dos Estados Unidos: https://www. ncbi.nlm.nih.gov/pmc/

- Europe PMC, mantido por associações de profissionais e instituições de saúde europeias: https://europepmc.org/

- Redalyc (Red de Revistas Científicas de América Latina y el Caribe, España y Portugal), o sistema de informação científica mantido pela Universidade Autônoma do Estado do México e por várias outras instituições de ensino e pesquisa (cerca de 550 periódicos): http://www.redalyc.org/ home.oa

- CNKI (China National Knowledge Infrastructure), acervo com cerca de dois mil periódicos da Universidade de Tsinghua e apoio dos Ministérios da Educação e da Ciência do governo chinês: http://oversea. cnki.net/kns55/default.aspx

A quarta medida de interesse para a internacionalização é a adoção do sistema administrativo ScholarOne Manuscripts, para gerenciar a submissão e a avaliação de manuscritos. Para as revistas integrantes da Coleção SciELO Brasil, esse sistema foi oferecido sem custo adicional; isto é, a expensas da SciELO. Mesmo assim, algumas revistas da coleção ainda não adotaram o sistema.

O ScholarOne apresenta muitas vantagens operacionais em relação aos antigos sistemas administrativos utilizados pelas revistas: permite a produção de estatísticas sobre o processo editorial e o controle de plágio e similaridade de texto, e faz sugestão automatizada de especialistas internacionais para indicação como revisores. O sistema ScholarOne tem ainda a vantagem de fazer em inglês a comunicação entre editores, autores e revisores, permitindo que a submissão de artigos, seu processamento editorial e a solicitação de pareceres sejam acessíveis para interessados que não falam português.

Por fim, a quinta medida para a difusão internacional dos periódicos de saúde coletiva editados no Brasil também é promovida pela Coleção SciELO Brasil: a adoção da licença de direitos de cópia Creative Commons (CC-BY). Essa opção autoriza redistribuir os artigos publicados em qualquer suporte ou formato, como os repositórios internacionais acima mencionados. Os artigos completos que foram publicados sob essa licença podem ser republicados integralmente ou em parte em livros ou coletâneas, desde que devidamente referenciados. Podem ser oferecidos nas redes de relacionamento de pesquisa e nos repositórios de pesquisa. Os autores podem carregar os arquivos PDF dos artigos publicados, no formato original da publicação, em sites como researchgate.net e academia.edu, em blogs e em páginas pessoais. $\mathrm{O}$ mesmo pode ser feito pelas instituições de ensino e pesquisa em seus repositórios institucionais, como revistas.usp.br, periodicos.fiocruz.br e arca.fiocruz.br. Todos esses recursos são de interesse das revistas de saúde coletiva e favorecem a divulgação internacional dos artigos publicados.

\section{O caminho que rejeitamos e as soluções que esperamos}

As revistas de saúde coletiva não rejeitam a internacionalização. Ao contrário, valorizam essa perspectiva, e vêm tomando medidas importantes nessa direção. Há interesse internacional pelo conhecimento produzido no País; há reconhecimento internacional quanto à qualidade da pesquisa aqui realizada e dos pesquisadores que aqui atuam. 
Mas o meio editorial da saúde coletiva resiste à adoção de critérios de avaliação da qualidade dos periódicos com base na contabilidade da participação de profissionais sediados no exterior como autores dos artigos publicados, revisores (peer review) e editores das revistas. Critérios desse tipo têm sido impostos unilateralmente em processos de avaliação em ciência e tecnologia, com resultados ainda obscuros.

Mas a avaliação induz o planejamento e a ação. Atribuir valor ao aumento de autores estrangeiros no portfólio de artigos publicados pode levar a distorções como induzir a aprovação de artigos de pior qualidade. Até mesmo em função do progresso editorial das revistas brasileiras, há uma crescente submissão de artigos de baixa qualidade produzidos no exterior, sem nenhuma perspectiva de interlocução com a pesquisa realizada no País. Autores estrangeiros eventualmente incluíram as revistas brasileiras no rol de oportunidades para publicação internacional de seus trabalhos, especialmente porque, em sua maioria, elas oferecem publicação em acesso aberto sem custo. É uma opção legítima por parte dos autores; no entanto, os editores precisam preservar sua autonomia para avaliar tais submissões com o mesmo critério que utilizam para avaliar os trabalhos produzidos no País. Em resumo, os artigos submetidos não devem ser priorizados em função de sua origem.

Valorizar mais a publicação de artigos de autores 'estrangeiros' (ou sediados no exterior) em relação aos autores 'nacionais' (ou sediados no País) rompe a premissa de que o processo de avaliação editorial deve ser autônomo e livre de constrangimentos extracientíficos. $\mathrm{O}$ mesmo pode ser dito quanto à seleção dos revisores e editores envolvidos no processo de avaliação de cada artigo. Os critérios para a seleção de editores e revisores devem ser a competência para avaliar os temas abordados e a independência entre avaliadores e avaliados. Valorizar mais a participação de avaliadores estrangeiros em relação aos nacionais também rompe a autonomia do processo editorial.
A credibilidade e o prestígio das revistas se fundamentam na qualidade desse processo. Revistas boas são aquelas que atraem os melhores artigos, e os selecionam e aprimoram por meio de peer review. Os periódicos científicos devem preservar a escolha, a avaliação e a edição dos artigos livres de interferências externas quanto a quem é escolhido para participar desses processos, ou quanto a seus resultados. A reflexão científica que seleciona e aprimora os artigos publicados deve estar restrita ao conselho editorial das revistas, a editores científicos e associados, e a revisores convidados para participar do processo.

O Brasil conta com profissionais com excelente qualificação para a interlocução internacional. Muitos brasileiros e estrangeiros que atuam nas instituições de ensino e pesquisa do País participam do processo editorial internacional como editores científicos, associados e revisores convidados das revistas estrangeiras, ou mesmo como autores de artigos publicados nessas revistas. Isto é, há prestígio e competência reconhecidos internacionalmente nos profissionais que aqui atuam.

Não é correto e não se justifica impor a participação de autores, revisores e editores sediados em instituições no exterior nos processos editoriais das revistas brasileiras. Quando se dá pela livre iniciativa dos profissionais e dos periódicos, essa participação pode ser proveitosa para a produção e a veiculação do conhecimento científico. Sob o pretexto de promover a internacionalização das revistas, a imposição de critérios de avaliação dos periódicos pelo país de origem dos autores, revisores e editores parece querer induzir um desnecessário e indesejado efeito de 'estrangeirização' dos periódicos produzidos no País.

Por fim, retomam-se dois pontos do que foi dito na introdução. Em primeiro, como foi constatado por Adams $\mathbf{1}$, ex-diretor de avaliação de pesquisa da Thomson Reuters e atual consultor da Universidade de Leeds, no Reino Unido, a melhor investigação é fruto da colaboração entre pessoas de vários países. Esse é o ponto alto da internacionalização, e 
depende de política científica. O País precisa ter incentivo para que pesquisadores e estudantes possam ir para o exterior, e para atrair colaboradores para as investigações locais. Isso é importante porque, pelo menos na área da saúde, salvo raras exceções, a participação dos brasileiros nos artigos de estrangeiros - quase sempre os americanos - não se dá com autores principais. Nesse sentido, as revistas estão na ponta extrema do processo científico: elas publicam o que foi produzido e não podem ser responsabilizadas pela ausência de cooperação entre pesquisadores.

O segundo ponto é sobre política científica. A divulgação feita pelos periódicos precisa fazer parte de uma política de governo que reconheça a importância da produção do conhecimento e a inclua em seu planejamento e em seu financiamento. $\mathrm{O}$ esforço de produzir uma revista e todas as estratégias para internacionalizá-la não podem ser responsabilidade apenas dos editores e dos autores. Portanto, é fundamental que a saúde pública/saúde coletiva, ao lado de outras áreas, provoque uma mudança de cultura no Ministério de Ciência e Tecnologia e nas instituições de fomento, para que sejam criadas fontes sustentáveis de financiamento e de internacionalização para as revistas brasileiras de reconhecido mérito.

\section{Colaboradores}

Antunes JLF (0000-0003-3972-9723)*, Barros AJD (0000-0002-2022-8729)* e Minayo MCS (0000-0001-6187-9301)* contribuíram igualmente na elaboração do manuscrito.

\section{Referências}

1. Adams J. The fourth age of research. Nature 2013; 497:557-560.

2. Carvalho MS, Travassos C, Coeli CM. A internacionalização da ciência. Cad. Saúde Pública 2014; 30(8):15851587.

3. Cunha-Melo JR. Indicadores efetivos da internacionalização da ciência. Rev Col Bras Cir 2015; 42(supl1):2025 .

4. Farias AS. Internacionalização dos periódicos brasileiros. Rev Adm Empres. 2017; 57(4):401-4.
5. Oliveira PHFD. Propagador das Sciencias Medicas e Semanário de Saúde Pública: as moléstias mentais nos periódicos médicos brasileiros (Rio de Janeiro, 1827-1833). Revista Discente do Programa de Pós-Graduação em História - UFJF. 2017; 3(6):92-109.

6. Monteiro CA, Barata RB, Antunes JLF. Cinquenta anos da Revista de Saúde Pública. Rev Saude Publica. 2016; 50:1.

7. Amarante P, Rizzotto MLF, Costa AM. Memória de um movimento: a revista Saúde em Debate e a reforma sanitária brasileira. Ciênc. Saúde Colet. 2015;
${ }^{*}$ Orcid (Open Researcher and Contributor ID). 
20(7):2023-2029.

8. Carvalho MS, Coeli CM, Travassos C. Uma breve história de Cadernos de Saúde Pública. Ciênc. Saúde Colet. 2015; 20(7):2007-2012.

9. Garcia LP, Duarte E. Epidemiologia e Serviços de Saúde: a trajetória da revista do Sistema Único de Saúde do Brasil. Ciênc. Saúde Colet. 2015; 20(7):2081-2090.

10. Minayo MCS, Gomes R. Ciência \& Saúde Coletiva no contexto nacional e internacional da divulgação científica. Ciênc. Saúde Colet. 2015; 20(7):2013-2022.
11. Almeida MF, Goldbaum M, Carvalheiro JR. A Revista Brasileira de Epidemiologia: 18 anos de contribuição à difusão de conhecimentos. Ciênc. Saúde Colet. 2015; 20(7):2031-2039.

12. Antunes JLF, Garcia LP, Almeida MF. O Fórum de Editores e a estratégia para ações políticas e científicas. Ensaios \& Diálogos em Saúde Coletiva 2017; 4:30-32.

Recebido em 08/01/2019 Aprovado em 10/05/2019

Conflito de interesses: inexistente

Suporte financeiro: não houve 